\title{
THE EFFECTS OF SURFACE ROUGHNESS ON PARTICLE DISPERSION, MIGRATION, AND SELF-DIFFUSIVITY IN LINEAR AND NONLINEAR SHEAR FLOWS
}

\author{
M. DARBEHESHTI, J. STOKAN, U. DAM \& M.S. INGBER \\ Department of Mechanical Engineering, University of Colorado Denver, Denver, CO, USA.
}

\begin{abstract}
Particle interactions in highly-viscous nonlinear and linear shear flows play an important role in a variety of applications including composite materials processing, microfluidics, chromatography, and particle resuspension, to name a few. Binary interactions among particles can provide information used in rheological models for suspension flows such as migration rates and self-diffusivity. In past numerical studies, particle roughness has been treated, for the most part, as a constant, static quantity. In the current study, roughness is treated as a stochastic parameter. Hence, quantities such as dispersion, net particle migration, and self-diffusivity also become stochastic parameters. Numerical simulations are performed using a semi-analytic solution for the motion of two particles in an arbitrary unbounded flow field to determine the effects of random particle roughness.

Keywords: particle dispersion, particle interactions, particle migration, self-diffusivity, suspension flows.
\end{abstract}

\section{INTRODUCTION}

Interactions of smooth particles in isothermal, Stokes flow are theoretically reversible. However, irreversibility can be introduced in a variety of ways including viscosity perturbations, inter-particle static forces, solvation forces, and particle roughness. In particular, particle roughness in suspension flows has been shown to cause asymmetries in particle trajectories in linear shear flows [1,2], particle pair migration [2] in nonlinear shear flows, and asymmetric pair distribution functions [3] in low-concentration suspension flows.

Experiments performed by Smart and Leighton [4] and Heath et al. [5] have shown that the magnitude of particle roughness can, in certain cases, be determined by measuring the difference in the time it takes for a particle to sediment towards a plane through a given distance, inverting the plane, and then measuring the time it takes the particle to sediment the same distance away from the plane. However, in the study by Smart and Leighton, particle roughness was made somewhat uniform by gluing sieved sand particles of relatively uniform size to the outside of larger spheres. In practice, roughness is not particularly uniform.

Popova et al. [6] performed experiments with three different types of PMMA particles in terms of the surface treatment, namely, original surface (relatively) smooth particles, lapped particles, and bead-blasted particles. They showed in their experiments conducted in a Couette device that particle migration scaled essentially with the square root of the rootmean-square particle roughness.

Ingber et al. [7] performed both experiments and numerical simulations of five particles in a Couette device. In the simulations, a traction-corrected boundary element method was employed with a static surface roughness model. They determined that the increase in the so-called particle radial moment matched in the average over the 52 experiments and corresponding numerical simulations when the imposed surface roughness in the numerical simulations was approximately four times the average physical particle roughness. 
For the most part, numerical simulations of binary sphere interactions in shear flow have treated roughness as a static quantity [2, 8, 9]. Zhao and Davis [10] considered the interaction of sedimenting spheres with essentially two levels of surface roughness. In general, surface roughness is a stochastic quantity. In this research, the effects of stochastic surface roughness on particle dispersion, particle migration, and particle self-diffusivity are studied.

\section{SIMULATION METHODOLOGY}

The problem under consideration is the binary interaction of two rough particles in the zero-Reynolds-number limit. The governing equation for the fluid is the Stokes equation and the governing equations for the particles are the equilibrium and kinematic equations. The simulation method used to perform the simulations in the current research is based on a semi-analytic solution for the motion of two spheres suspended in an unbounded but otherwise arbitrary shear flow [11]. The semi-analytic method is a vast generalization of classical bispherical-coordinate solutions for two spheres moving along or perpendicular to their line of centers, rotating about the centerline in a quiescent liquid, or suspended in linear shear flow. The method is highly efficient and very convenient since the algorithm does not require any local expansions of the ambient velocity field $\mathrm{u}(\mathrm{x})$, but simply operates with the values of $\mathrm{u}(\mathrm{x})$ in the vicinity of the spheres, which can be calculated either by an analytic formula (e.g., for Poiseuille flow) or by a user-provided routine for more complex cases. The method provides linear and angular velocities as a function of position. Once the linear and angular velocities are determined, the particles are repositioned in space using a third-order, variable-time-step Runge-Kutta routine. The governing equations considered in this research are reversible. Further, this particular solution algorithm is extremely accurate, and hence, the particle motions for presumed smooth spheres are essentially reversible. The roughness model considered in this research is essentially equivalent to the non-locked model of DaCunha and Hinch [8] in which particles are not allowed to have separations less than a specified roughness, e, but are allowed to rotate relative to each other. However, unlike previous research $[2,8]$, roughness is presumed to be a random stochastic quantity modeled as a normal distribution with specified average roughness and standard deviation.

\section{RESULTS}

We consider the interaction of two rough spheres in both linear and nonlinear shear (Poiseuille) flow. For the case of Poiseuille flow, the far-field velocity profile is in the $x$-direction and is given by

$$
u=b-c(z-d)^{2}
$$

where $b, c$, and $d$ are constants. The average sphere radius is denoted by a. The following definitions are useful in characterizing these problems. The coordinates of the two particles are given by $\left(x_{1}, y_{1}, z_{1}\right)$ and $\left(x_{2}, y_{2}, z_{2}\right)$. The initial center to center separations of the spheres in the three coordinate directions are given by $\Delta x_{-\infty}, \Delta y_{-\infty}$, and $\Delta z_{-\infty}$. The shear plane is the $x-z$ plane, the far-field velocity is in the $x$-direction, and the initial separation is given by $\Delta x_{-\infty} / a=-10$. The simulations are stopped when $\mathrm{x}_{2}-\mathrm{x}_{1}=10 \mathrm{a}$ which then sets the downstream separations denoted by $\Delta x_{\infty}, \Delta y_{\infty}$, and $\Delta z_{\infty}$. The particle trajectories are typically drawn with respect to the transient separation $\Delta x=x_{1}-x_{2}$.

Typical transient trajectories of the particle pairs in the shear plane are shown in Fig. 1 for the case $\Delta z_{-\infty} / a=0.2, \Delta y_{-\infty} / a=0.0, b=41 / 40, c=5 / 8, d=1 / 2$, and particle roughness ranging between $\varepsilon / a=0=0.01$. As seen in Fig. 1 for the case $\varepsilon / a=0.0$ (smooth spheres), 


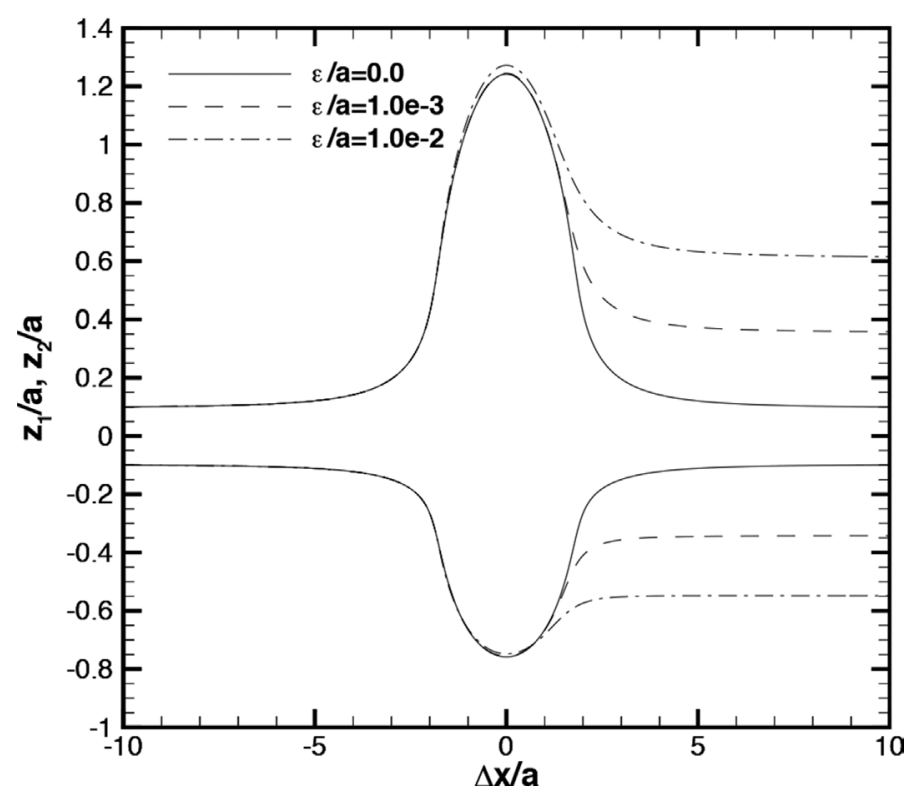

Figure 1: Particle trajectories in the shear plane of two rough spheres suspended in Poiseuille flow with initial positions $(-5.0,-0.05,0.1)$ and $(5.0,0.05,-0.1)$, $\Delta y_{-\infty} / a=0.0$ and $\Delta z_{-\infty} / a=0.2$.

the particle trajectories are symmetric about $\Delta x / a=0$ and, in particular, $\Delta z_{\infty}=\Delta z_{-\infty}$. This indicates that the particle trajectories are essentially reversible. As the roughness increases, the in-plane particle dispersion $\Delta z_{\infty}-\Delta z_{-\infty}=\Delta z_{ \pm \infty}$ is also seen to increase indicating increasing irreversibility in the system with increasing particle roughness. Another quantity of interest is the net particle migration which is the location of the center of mass of the particle pair, $z_{\mathrm{cm}}$, at the end of the simulation. Again, for smooth particles, there is no net particle migration. As the particle roughness increases, the net particle migration also increases with $z_{c m}>0$ indicating that the particle pair has migrated towards the low-shear-rate region of the flow field.

In this paper, we focus on two measures of irreversibility defined in the previous paragraph, namely, particle dispersion and particle migration. The dispersion is essentially a measure of the difference of the final lateral separation of the spheres minus the initial lateral separation. The particle migration measures the net motion of the center of mass of the sphere pair relative to the high- or low-shear rate region of the ambient nonlinear flow field. For reversible flows, there is no particle dispersion or migration. For irreversible flows, both quantities are stochastic for stochastic particle roughness.

The analysis of in-plane particle dispersion and migration in previous studies [2, 11] has assumed that the particle roughness is constant. The effect of stochastic surface roughness on particle dispersion and net particle migration over 20 simulations is shown in Fig. 2 again for the case $\Delta z-\infty / a=0.2, \Delta y_{-\infty} / a=0.0, b=41 / 40, c=5 / 8$, and $d=1 / 2$. As seen in Fig. 2, both particle dispersion and migration correlate essentially perfectly with the stochastic particle roughness which is not surprising considering roughness is the only 
form of irreversibility introduced into the flow. (There is some very small irreversibility introduced into the simulations caused by numerical error, but this irreversibility is imperceptible in Fig. 2.)

A quantity of interest is the so-called nonlinearity parameter, $N L P$, defined by

$$
N L P=\frac{a \nabla \dot{\gamma}}{\dot{\gamma}+\dot{\gamma}_{N L}}
$$

where $a$ is the sphere radius, $\dot{\gamma}$ is the local shear rate calculated at the initial location of the center of mass of the particle pair, and $\dot{\gamma}_{N L}$ is the so-called nonlocal contribution to the shear rate ([12]). The nonlocal shear rate is given by

$$
\dot{\gamma}_{N L}=\frac{a}{D} \dot{\gamma}_{o}
$$

where $D$ is the distance between the "walls" and $\dot{\gamma}_{o}$ is the shear rate for the unperturbed flow at the "wall". In the current simulations since the flow field is unbounded, the distance between the walls is taken as the distance between the two locations where the velocity of the ambient flow field is zero. As the name suggests, the nonlinearity parameter is a measure of the nonlinearity of the flow field. The effect of the nonlinearity parameter on the standard deviation of the net migration is shown in Fig. 3 for three different values of roughness standard deviation for an initial separation given by $\Delta z_{-\infty} / a=0.2, \Delta y_{-\infty} / a=0.0$, and average sphere roughness given by $\varepsilon / a=0.001$. As seen in Fig. 3, the standard deviation of the net migration increases with both increasing nonlinearity in the flow field and increasing the standard deviation of the sphere roughness.

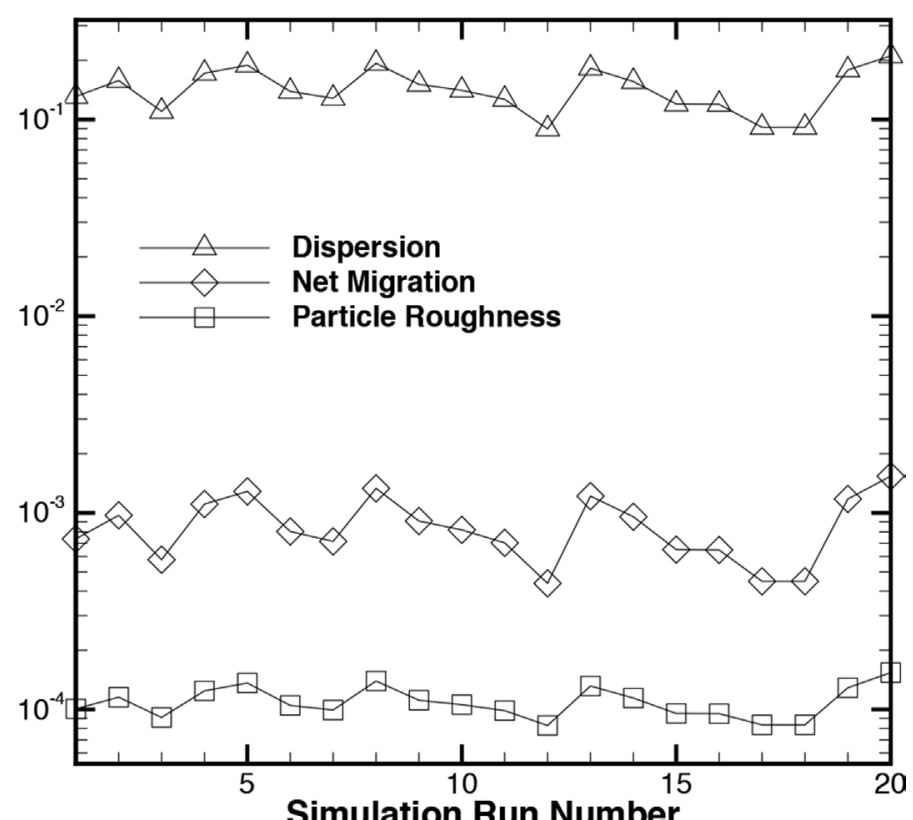

Figure 2: Dispersion, migration, and particle roughness for 20 simulations for an average roughness of $\varepsilon / a=1.0 e-4$ and standard deviation of $\sigma=2.0 e-5$. 


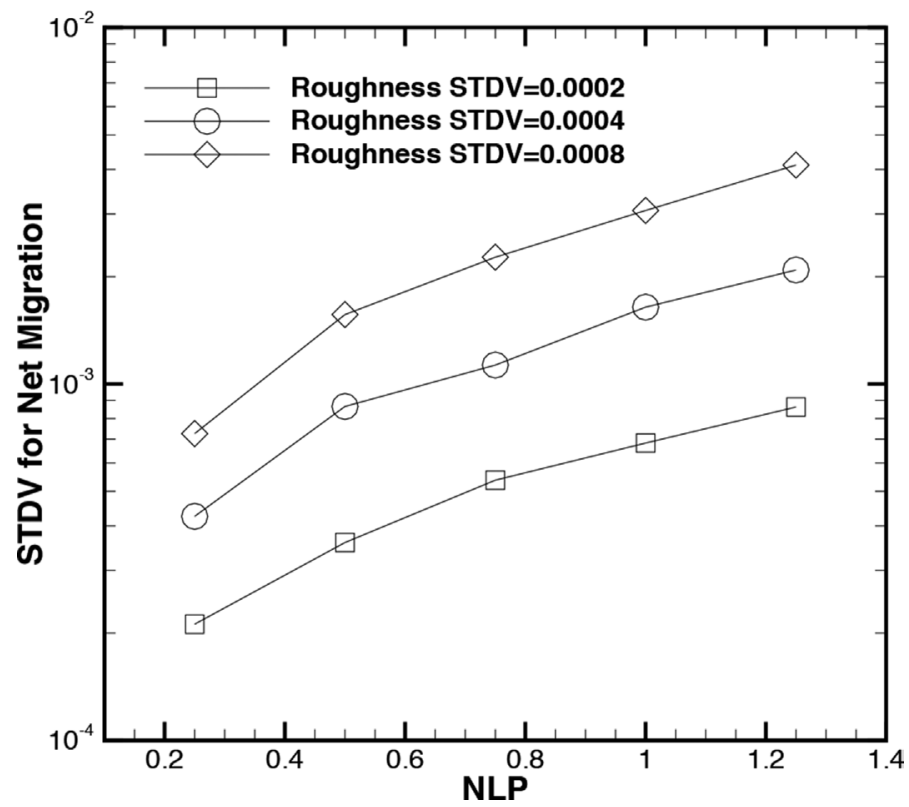

Figure 3: Standard deviation, STDV, of the particle net migration as a function of the nonlinearity parameter, $N L P$ (eqn (3)), for the case $\Delta z_{-\infty} / a=0.2$, $\Delta y_{-\infty} / a=0.0$, and $\varepsilon / a=1.0 e-3$.

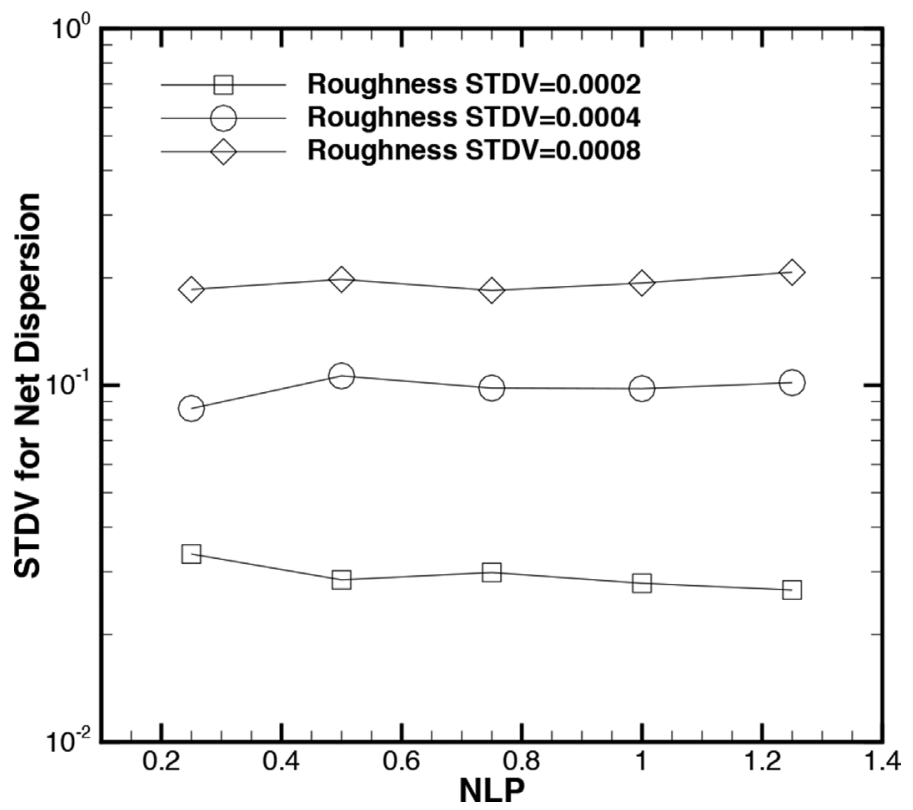

Figure 4: Standard deviation, STDV, of the particle dispersion as a function of the nonlinear-ity parameter, $N L P$ (eqn (3)), for the case $\Delta z_{-\infty} / a=0.2$, $\Delta y_{-\infty} / a=0.0$, and $\varepsilon / a=1.0 e-3$. 
The dispersion, $\Delta z \pm \infty$, as a function of the nonlinearity parameter is shown in Fig. 4. As seen in Fig. 4, there is little influence of the nonlinearity parameter on the dispersion. This is actually an expected result as it has previously been shown $[2,11]$ that the nonlinearity parameter has little influence on particle self-diffusivity, which is primarily a function of particle dispersion as discussed below.

One of the key relationships of interest is the effect of uncertainties in surface roughness on both dispersion and net particle migration as characterized by the relationship between the standard deviation of surface roughness with the standard deviation of dispersion and the standard deviation of net particle migration. These relationships are shown in Figs 5 and 6, respectively. As seen in Fig. 5, the nonlinearity parameter has little effect on the standard deviation of the dispersion. On the other hand as seen in Fig. 6, the standard deviation of the net particle migration increases monotonically with increasing nonlinearity of the flow field.

Even though there is increasing uncertainty in both the dispersion and net particle migration in these binary particle interactions, the two quantities correlate almost perfectly as shown in Fig. 7 for the case of average particle roughness $\varepsilon / a=1.0 e-3$ and particle standard deviation given by $\sigma_{p}=2.0 e-4$. For each nonlinearity parameter, twenty simulations are run each with a random particle roughness. The correlation emphatically demonstrates that both particle dispersion and migration are a direct result of irreversibility within the fluid-particle system. Essentially, with an increase in particle roughness (irreversibility) both the particle dispersion and migration increase in a predictable manner.

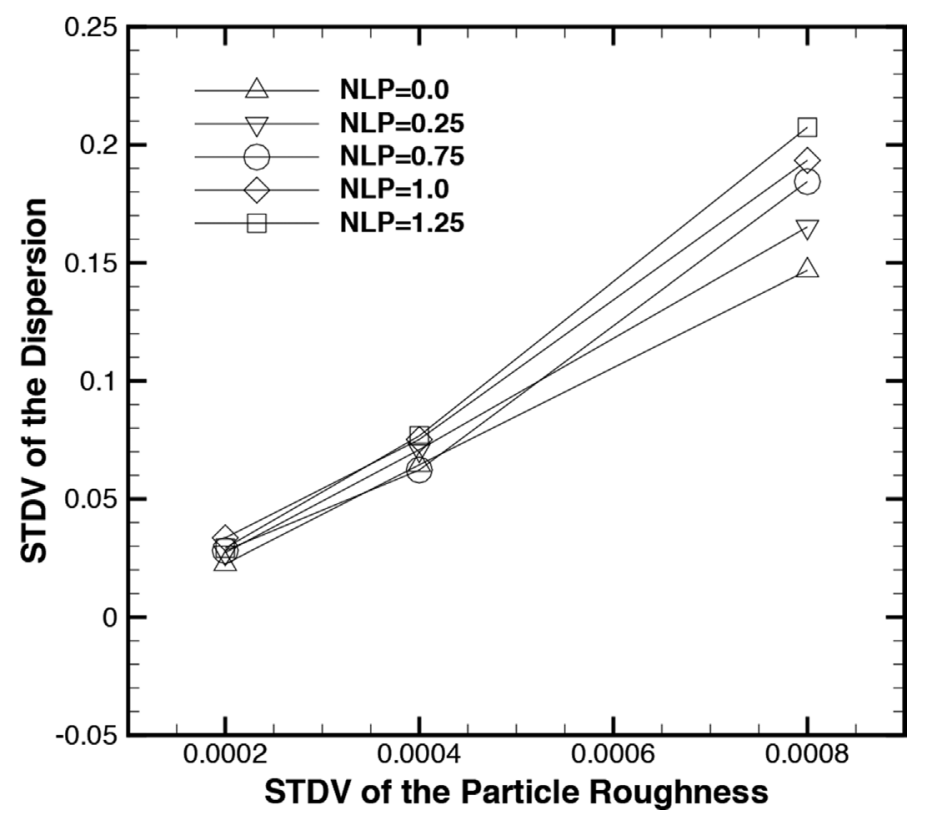

Figure 5: The standard deviation, STDV, of the particle dispersion as a function of the standard deviation of the particle roughness over a range of nonlinearity parameters (eqn (3)) for the case $\Delta z_{-\infty} / a=0.2, \Delta y_{-\infty} / a=0.0$, and $\varepsilon / a=1.0 e-3$.. 


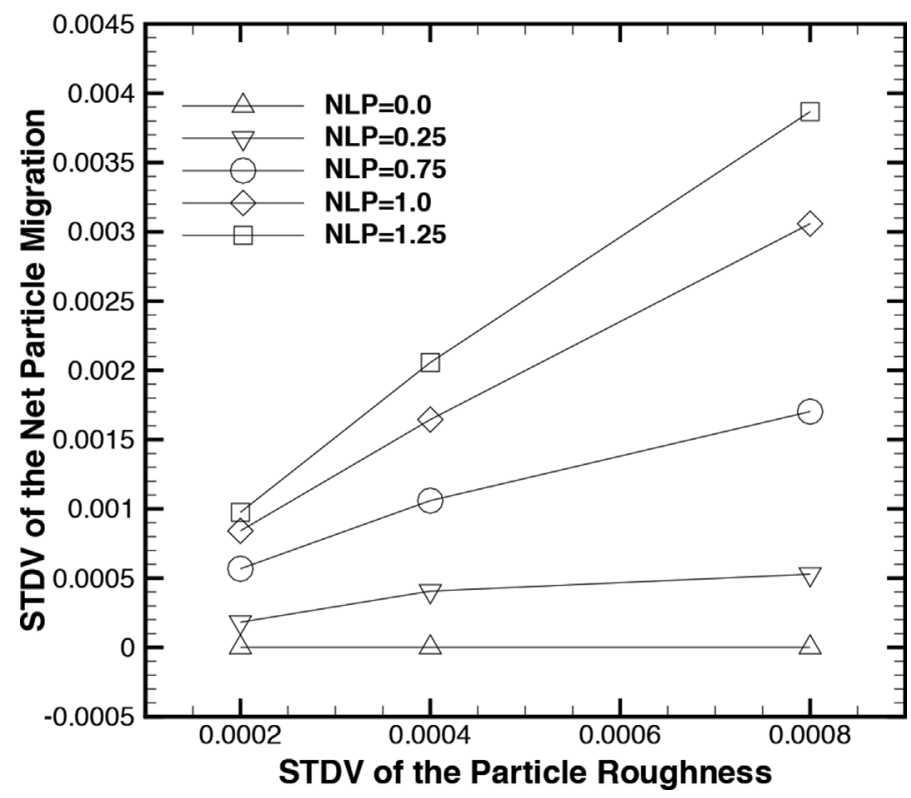

Figure 6: The standard deviation, STDV, of the net particle migration as a function of the standard deviation of the particle roughness over a range of nonlinearity parameters (eqn (3)) for the case $\Delta z_{-\infty} / a=0.2, \Delta y_{-\infty} / a=0.0$, and $\varepsilon / a=1.0 e-3$.

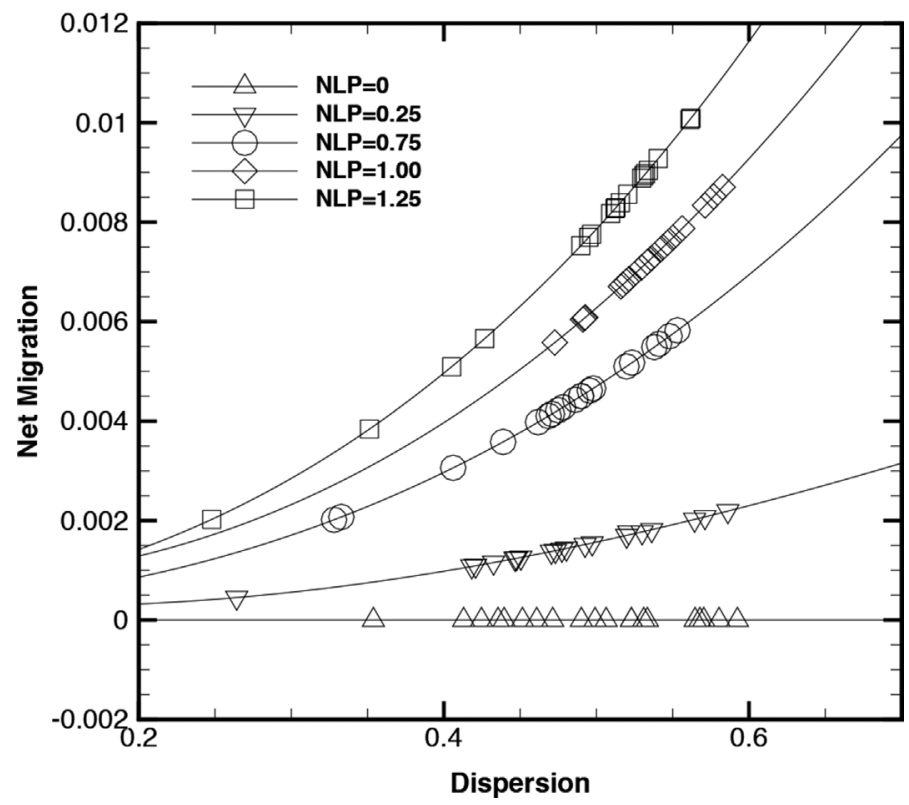

Figure 7: Dispersion versus net particle migration 20 simulations for an average particle roughness of $\varepsilon / a=1.0 e-3$ and particle standard deviation of $\sigma_{p}=2.0 e-4$ 
The final quantities to be considered in this research are the in- and out-of-plane selfdiffusivities of binary sphere interactions. The in-plane and out-of-plane self-diffusivities in the shear plane, $D_{z}^{s}$ and $D_{y}^{s}$, respectively, can be calculated by evaluating the following integrals [2].

$$
\begin{aligned}
& D_{z}^{s}=\phi a^{2} \dot{\gamma} \frac{3}{8 \pi} \int_{-\infty}^{\infty} \int_{-\infty}^{\infty}\left(\Delta z_{ \pm \infty}\right)^{2} \Delta z_{-\infty} d y_{-\infty} d z_{-\infty} \\
& D_{z}^{s}=\phi a^{2} \dot{\gamma} \frac{3}{8 \pi} \int_{-\infty}^{\infty} \int_{-\infty}^{\infty}\left(\Delta y_{ \pm \infty}\right)^{2} \Delta z_{-\infty} d y_{-\infty} d z_{-\infty}
\end{aligned}
$$

Both the in-plane and out-of-plane self-diffusivities are caused by particle roughness. In all previous simulations considered in this paper, the particle centers have been in the shear plane, and hence, there has been no out-of-plane dispersion caused by particle roughness. If the particle centers are not both in the shearplane, there will be out-of-plane dispersion, which will result in out-of-plane self-diffusivity.

The in-plane self-diffusivity, $D_{z}^{s}$, for linear shear flow over 20 different simulations with the average particle roughness of $\varepsilon / a=0.001$ is shown in Fig. 8 for three different standard deviations of the roughness. As seen in Fig. 8, the average in-plane self-diffusivity does not change appreciably with roughness standard deviation. In fact, the average in-plane self-diffusivity, $\bar{D}_{z}^{s}$, is given by $7.91 \mathrm{e}-4,7.82 \mathrm{e}-4,7.86 \mathrm{e}-4$ for roughness standard deviations of $0.0002,0.0004$, and 0.0008 , respectively. Similarly, the standard deviation of the in-plane self-diffusivity is given by $1.342 \mathrm{e}-5,2.418 \mathrm{e}-5$, and $3.279 \mathrm{e}-5$ again for roughness standard deviations of 0.0002 , 0.0004 , and 0.0008 respectively, showing a monotonic increase in the standard deviation of the in-plane self-diffusivity with increasing standard deviation of the particle roughness.

The average in-plane and out-of-plane self-diffusivities are shown in Figs 9 and 10, respectively, as a function of the particle roughness standard deviation over a range of nonlinearity

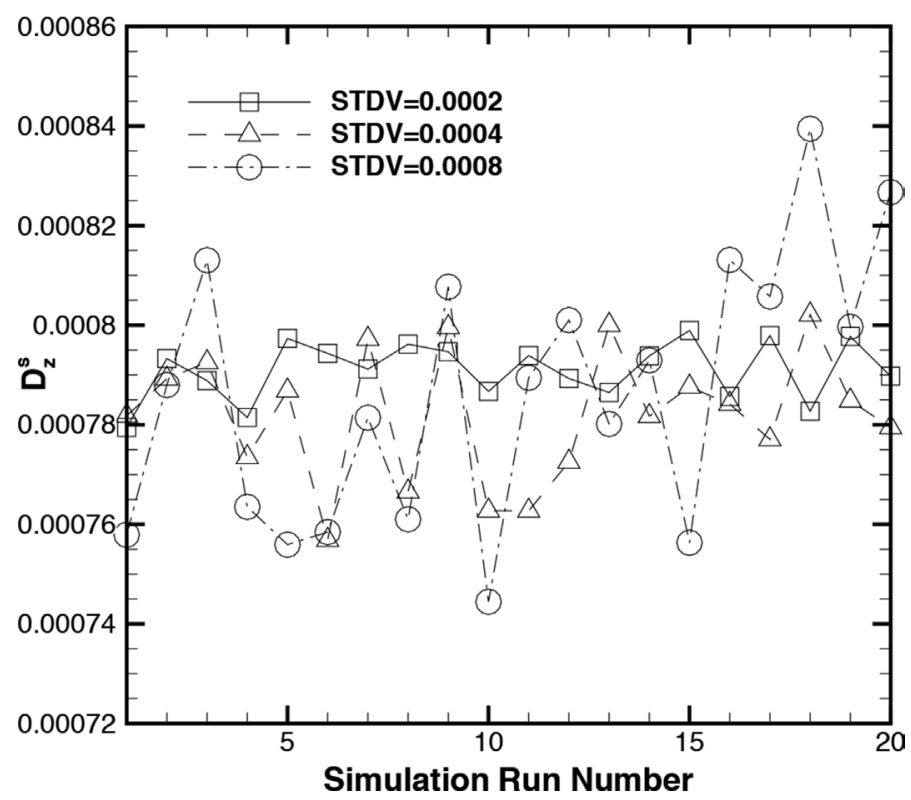

Figure 8: In-plane self-diffusivity $D_{z}^{s}$ for a linear shear flow for an average particle roughness given by $\varepsilon / a-0.001$. 


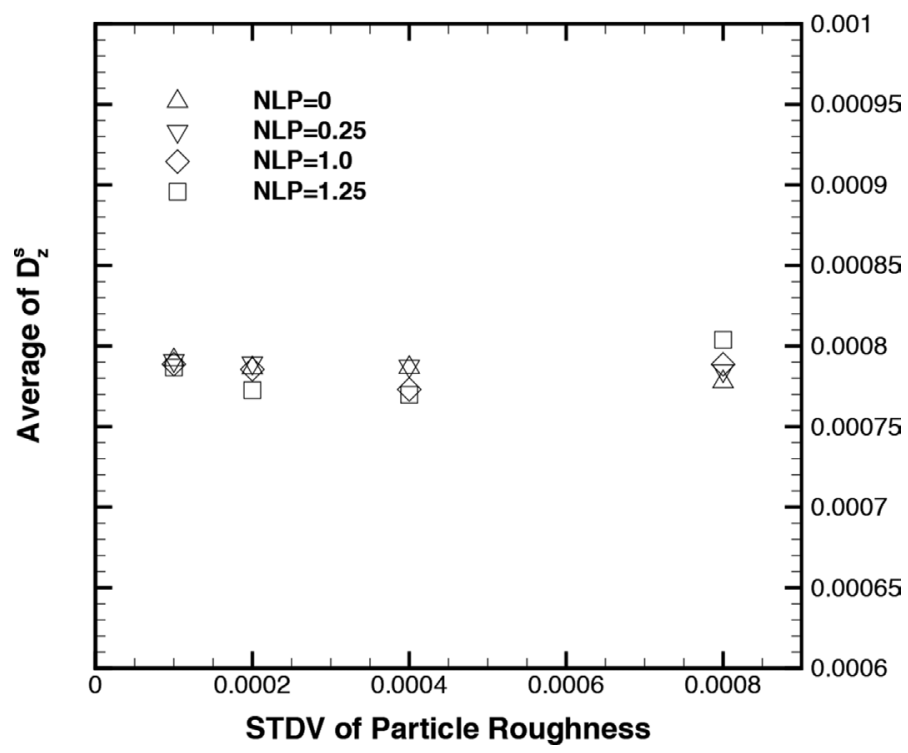

Figure 9: Average in-plane self-diffusivity, $D_{z}^{s}$, for the case $\varepsilon / a-0.001$.

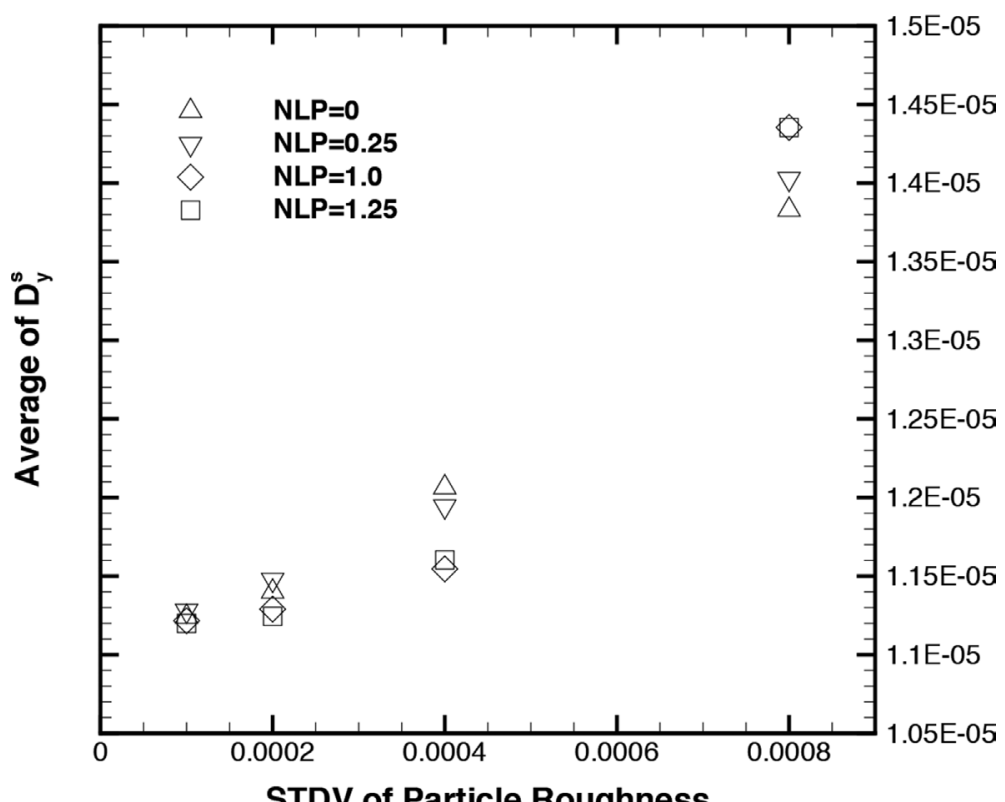

Figure 10: Average out-of-plane self-diffusivity, $D_{z}^{s}$, for the case $\varepsilon / a-0.001$.

parameters for the case $\varepsilon / a=0.001$. The average was calculated over 20 simulations. As seen in Fig. 9, the average in-plane self-diffusivity does not depend either on the nonlinearity parameter nor the standard deviation of the particle roughness. As discussed earlier, the nonlinearity of the flow field does not influence the dispersion, and hence, it is not surprising 
that it has little effect on the self-diffusivity. As seen in Fig. 10, the nonlinearity of the flow field again has little effect on the out-of-plane self-diffusivity. However, unlike the case of the in-plane self-diffusivity, the average out-of-plane self-diffusivity is seen to increase with an increasing standard deviation of the particle roughness. It can be conjectured that this is a result of the larger particle roughness experienced with larger standard deviations having a disproportionate effect on the self-diffusivity. This may also be true to a lesser extent for the

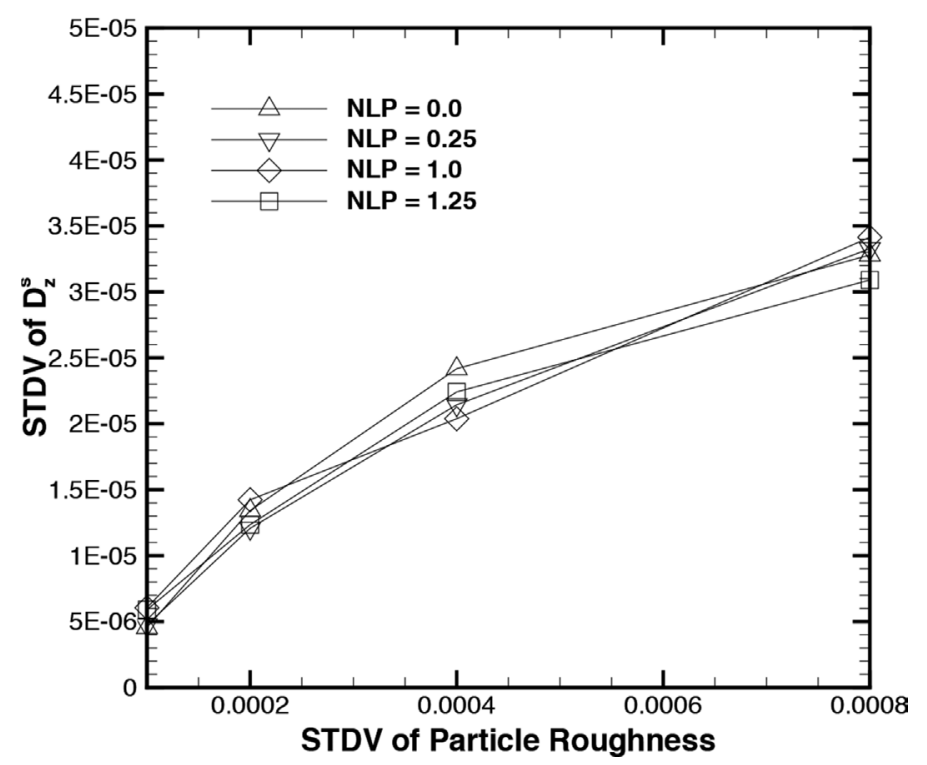

Figure 11: Standard deviation of the in-plane self-diffusivity for the case $\varepsilon / a-0.001$.

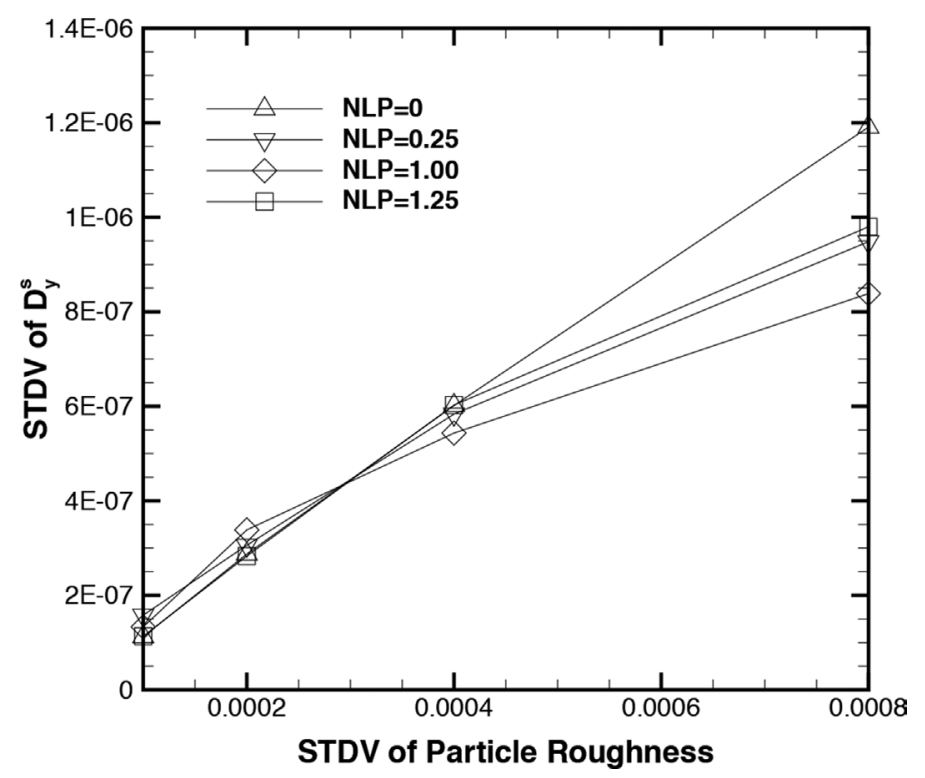

Figure 12: Standard deviation of the out-of-plane self-diffusivity for the case $\varepsilon / a-0.001$. 
in-plane self-diffusivity, but may not be noticeable since the out-of-plane self-diffusivity is an order of magnitude less than the in-plane self-diffusivity.

The standard deviation of the in-plane and out-of-plane self-diffusivities are shown in Figs 11 and 12, respectively, as a function of the particle roughness standard deviation over a range of nonlinearity parameters for the case $\varepsilon / a=0.001$. It is seen in Figs 11 and 12 that the nonlinearity of the flow has little influence on the standard deviation of the self-diffusivities. The standard deviation of the self-diffusivities increase with an increasing standard deviation of the particle roughness, but both the in- and out-of-plane curves are seen to be concave downwards. This may be a result of the fact that, as the standard deviation of the particle roughness increases, more of the simulations are run with a particle roughness of zero. The reason for this is that, if the random number generator which provides the normal distribution of particle roughness around the mean results in a negative roughness, the roughness in the simulation is set to zero. These cases are considered to be situations in which there is a crevice on the surface of the particle. The simulation does not allow the particles to interpenetrate, and hence, the particle roughness is reset from a negative value to zero. However, for each case of zero roughness, the simulation predicts zero dispersion, which reduces the standard deviation in the self-diffusivities.

\section{CONCLUSIONS}

The effects of stochastic surface roughness on the binary interactions of spheres in linear and nonlinear shear flow are considered in this research. Stochastic surface roughness causes parameters such as particle dispersion, migration, and self-diffusivity to also be stochastic parameters. There is essentially a perfect correlation between increases and decreases in the surface roughness with increases and decreases in particle-pair dispersion and net migration.

This correlation can be further buttressed by directly plotting particle migration versus dispersion over multiple simulations with random particle roughness. This result conclusively establishes that both particle dispersion and migration are caused by the only irreversibility included in the system, namely, particle roughness.

The standard deviation of the net particle migration increases with both increasing nonlinearity of the flow field and increasing standard deviation of the particle roughness. However, the standard deviation of the particle dispersion only increases with an increasing standard deviation of the particle roughness while the nonlinearity of the flow field has relatively little influence on this quantity. This is consistent with earlier results [2] which showed that the nonlinearity of the flow field did not affect particle dispersion appreciably.

The average in-plane self-diffusivity was essentially constant with an increasing standard deviation of the particle roughness. On the other hand, the average out-of-plane self-diffusivity increased with increasing standard deviation. It is conjectured that this is caused by large values of the particle roughness having a disproportionate effect of the out-of-plane self-diffusivity. Both the standard deviation of the in-plane and out-of-plane self-diffusivity increased with an increasing standard deviation of the particle roughness, but were essentially insensitive to the nonlinearity of the flow field. This insensitivity is expected since it was previously demonstrated that the nonlinearity of the flow field does not appreciably affect particle dispersion. The curves showing the standard deviations of in-plane and outof-plane dispersion as a function of the standard deviation of the particle roughness were both concave downwards. This is most likely a result of the fact that, as the standard deviation of the particle roughness increases, more and more simulations are run with a particle roughness of zero to preclude the particles from interpenetrating and hence reducing the scatter in the data. 


\section{ACKNOWLEDGMENT}

This material is based upon work supported by the National Science Foundation under Grant No. CBET-1335781. Any opinions, findings, and conclusions or recommendations expressed in this material are those of the authors and do not necessarily reflect the views of the National Science Foundation.

\section{REFERENCES}

[1] Zeng, S.L., Kerns, E.T. \& Davis, R.H., The nature of particle contacts in sedimentation. Physics of Fluids, 8(6), pp. 1389-1396, 1996. http://dx.doi.org/10.1063/1.868946

[2] Ingber, M.S., Feng, S., Graham, A.L. \& Brenner, H., The analysis of self-diffusion and migration of rough spheres in nonlinear shear flow using a traction-corrected boundary element method. Journal of Fluid Mechanics, 598, pp. 267-292, 2008. http://dx.doi.org/10.1017/S0022112007000043

[3] Rampall, I., Smart, J.R. \& Leighton, D.T., The influence of surface roughness on the particle-pair distribution function of dilute suspensions of non-colloidal spheres in simple shear flow. Journal of Fluid Mechanics, 339, pp. 1-24, 1997.

http://dx.doi.org/10.1017/S002211209600479X

[4] Smart, J.R. \& Leighton, D.T., Measurement of the hydrodynamic surface roughness of noncolloidal spheres. Physics Fluids A, 1, pp. 52-60, 1989. http://dx.doi.org/10.1063/1.857523

[5] Heath, C.E., Feng, S., Day, J.P., Graham, A.L. \& Ingber, M.S., Near contact interactions between a sphere and a plane. Physics Review E, 77, 2008. http://dx.doi.org/10.1103/physreve.77.026307

[6] Popova, M., Vorobieff, P. \& Graham, M.S.I.A.L., Interaction of two particles in a shear flow. Physics Review E, 75, 2007. http://dx.doi.org/10.1103/physreve.75.066309

[7] Ingber, M.S., Mammoli, A.A., Vorobieff, P., McCollum, T. \& Graham, A.L., Experimental and numerical analysis of irreversibilities particles suspended in a Couette device. Journal of Rheology, 50(2), pp. 99-114, 2006. http://dx.doi.org/10.1122/1.2169806

[8] DaCunha, F.R. \& Hinch, E.J., Shear-induced dispersion in a dilute suspension of rough spheres. Journal Fluid Mechanics, 309, pp. 211-223, 1996. http://dx.doi.org/10.1017/S0022112096001619

[9] Wilson, H.J. \& Davis, R.H., The viscosity of a dilute suspension of rough spheres. Journal Fluid Mechanics, 421, pp. 339-367, 2000. http://dx.doi.org/10.1017/S0022112000001695

[10] Zhao, Y. \& Davis, R.H., Interaction of sedimenting spheres with multiple surface roughness scales. Journal of Fluid Mechanics, 492, pp. 101-129, 2003. http://dx.doi.org/10.1017/S0022112003005652

[11] Ingber, M.S. \& Zinchenko, A., Semi-analytic solution of the motion of two spheres in arbitrary shear flow. International Journal of Multiphase Flow, 42, pp. 152-163, 2012. http://dx.doi.org/10.1016/j.ijmultiphaseflow.2012.01.005

[12] Miller, R.M. \& Morris, J.F., Normal stress-driven migration and axial development in pressure-driven flow of concentrated suspensions. Journal of Non-Newtonian Fluid Mechanics, 135, pp. 149-165, 2006.

http://dx.doi.org/10.1016/j.jnnfm.2005.11.009 